\title{
Schreiben des Hrn. Peano in Turin an die Redaction.
}

Dans l'interessante Note que M. Hočevar vient de publier dans votre journal t. V, p. 307, ${ }^{1}$ ) les premières propositions sont un cas particulier d'une formule contenue dans le Formulaire de Mathématiques (publié par la Rivista di Matematica) partie VII, \$. 1, prop. 5. Cette proposition est énoncée sous la forme

$$
\begin{gathered}
u \varepsilon K q . l^{\prime} u=\infty \cdot f \varepsilon q f u \cdot \nu \varepsilon K q \cdot v \diamond u . l^{\prime} \nu=\infty . \\
o . \lim _{x, v, \infty} f x, \lim _{x, u, \infty} f x
\end{gathered}
$$

qu'on lit: "Soit u une classe de nombres réels, dont la limite supérieure est infinie; soit $f$ la charactéristique d'une fonction réelle définie dans la classe $u$. Si v est un autre ensemble de nombres réels, contenu dans $u$, et dont la limite supérieure soit aussi l'infini, alors toutes les valeurs limites de la fonction $f x$, lorsque $x$, en variant dans l'ensemble v tend à l'infini, sont aussi des valeurs limites de la même fonction, lorsque $x$ tend à l'infini en variant dans la classe $u$."

Or si les valeurs limites dans le premier cas sont des valeurs limites dans le second cas, en resulte que les limites supérieure et inférieure des premières valeurs qu'on appelle les limites d'indetermination (Unbestimmtheitsgrenzen) de la fonction, sont comprises entre les limites correspondantes dans le deuxième cas, comme dit la prop. 10 du $\$$. 3 de la partie $V$ du même formulaire.

1) In der erwähnten Abhandlung blieben leider einige Druckfehler unberücksichtigt; so muss Seite 307 , Zeile 1 und 4 von unten, Seite 308 , Zeile 3 von oben und Seite 311, Zeile 3 von unten offenbar überall $O$ statt 0 stehen; Seite 312, Zeile 6 von unten hat zu lauten $-a_{2}+a_{1}-a_{4}+a_{3}-a_{6}+a_{5}-\ldots$ 